\title{
Microfluidic Particle Sorting in Concentrated Erythrocyte Suspensions
}

\author{
Stefan H. Holm, ${ }^{1}$ Zunmin Zhang, ${ }^{2}$ Jason P. Beech, ${ }^{1}$ Gerhard Gompper, ${ }^{2}$ Dmitry A. Fedosov, ${ }^{2, *}$ and \\ Jonas O. Tegenfeldt ${ }^{1, \dagger}$ \\ ${ }^{1}$ Department of Physics and NanoLund, Lund University, Lund, Sweden \\ ${ }^{2}$ Theoretical Soft Matter and Biophysics, Institute of Complex Systems and Institute for Advanced Simulation, \\ Forschungszentrum Jülich, 52425 Jülich, Germany
}

(Received 27 November 2018; revised manuscript received 10 March 2019; published 26 July 2019)

\begin{abstract}
An important step in diagnostics is the isolation of specific cells and microorganisms of interest from blood. Since such bioparticles are often present at very low concentrations, throughput needs to be as high as possible. In addition, to ensure simplicity, a minimum of sample preparation is important. Therefore, sorting schemes that function for whole blood are highly desirable. Deterministic lateral displacement (DLD) devices have proven to be very precise and versatile in terms of a wide range of sorting parameters. To better understand how DLD devices perform for blood as the hematocrit increases, we carry out measurements and simulations for spherical particles in the micrometer range which move through DLD arrays for different flow velocities and hematocrits ranging from pure buffer to concentrated erythrocyte suspensions mimicking whole blood. We find that the separation function of the DLD array is sustained even though the blood cells introduce a shift in the trajectories and a significant dispersion for particles whose diameters are close to the critical size in the device. Simulations qualitatively replicate our experimental observations and help us identify fundamental mechanisms for the effect of hematocrit on the performance of the DLD device.
\end{abstract}

DOI: 10.1103/PhysRevApplied.12.014051

\section{INTRODUCTION}

Blood analysis is an important procedure in biomedicine. As the blood sample drawn from a patient usually contains a plethora of biological markers, ranging from electrolytes, signaling molecules, proteins, and nucleic acids to viruses, cells, and microorganisms, an efficient sorting must be accomplished to minimize the confounding effects of irrelevant cells or to enrich the bioparticles of interest for early and accurate blood diagnosis. This is typically done using centrifugation, which is a simple and well-established technique. However, it requires large sample volumes, making it difficult to integrate with lab-on-a-chip devices.

To significantly decrease the amount of sample needed for detailed analysis, several microfluidic approaches have been developed [1-4]. For example, acoustophoresis [5,6] and inertial focusing $[7,8]$ offer high throughput but require

\footnotetext{
*d.fedosov@fz-juelich.de

†jonas.tegenfeldt@ftf.lth.se

Published by the American Physical Society under the terms of the Creative Commons Attribution 4.0 International license. Further distribution of this work must maintain attribution to the author(s) and the published article's title, journal citation, and DOI.
}

diluted samples. Particle margination or migration in blood flow is an interesting approach that mimics white blood cell (WBC) migration in small blood capillaries [9] and works best with red blood cell (RBC) hematocrits (volume fraction of RBCs) similar to those in whole blood [10]. The margination effect has also been used to separate out stiffer infected blood cells for malaria detection [11]. However, it is quite limited in scope.

Deterministic lateral displacement (DLD), on the other hand, has been shown to be a highly versatile tool for microfluidic sorting of spherical particles by size $[12,13]$. DLD devices utilize micropost arrays, which result in two different types of motion depending on the size of the particles: (i) zigzagging for small spherical particles with a nearly zero lateral motion and (ii) lateral displacement of large rigid particles along the array's structural lanes. The threshold size over which the particles move with lateral displacement is known as the critical size and depends on the device geometry. An approximate analytical expression has been derived [13]. However, recent studies $[14,15]$ show the existence of a mixed mode for the motion of rigid spherical particles in DLD devices, which has been explained by broken flow symmetry [16] and anisotropic permeability [14,17] in DLD arrays. These studies [16,17] also suggest how to better control the lateral displacement of spherical particles in DLD 
devices and to reliably estimate their corresponding critical sizes.

While the first studies using DLD devices have focused on the sorting of microspheres as a function of size, subsequent developments have demonstrated possibilities for sorting with respect to other relevant particle properties, for example, shape [18-22], deformability [2326], viscosity contrast [27], and dielectric properties [28]. Numerous research efforts have also considered the effects of different device geometries on sorting [15,19,29-31]. One of the general conclusions from the majority of such investigations is that even though the sorting of anisotropic and deformable particles in DLD devices is very promising, it is much more complex than the separation of rigid spherical particles by size. For instance, high shear stresses at high flow rates may significantly deform soft particles, changing their effective size $[3,23,25]$. Furthermore, particle deformation and dynamics may affect the particle's traversal through a DLD device [18,27].

The first separations performed on blood [3] showed the relevance of DLD devices for diagnostics. Subsequent studies have demonstrated the sorting of many biologically and diagnostically relevant components under strongly diluted blood conditions [20,30,32,33]. A question that remains largely unexplored is the effect of blood hematocrit on the performance for sorting and detection of particles suspended in blood. Successful and reliable separation of particles from whole blood has only been achieved for large cells, such as WBCs [34,35] and circulating tumor cells (CTCs) [36-39]. This is possible because the targeted cells are significantly larger than RBCs, which do not appear to strongly disturb the trajectories of WBCs and CTCs. However, for smaller cells and microorganisms (e.g., bacteria) in blood, the concern remains that nondeterministic interactions between them and RBCs will affect their trajectories and have deleterious effects on sorting. For instance, simulations of RBCs at high concentrations in three dimensions have shown that the trajectories of RBCs in DLD devices are significantly affected by finite hematocrit [40]. To optimize sorting of particles from concentrated erythrocyte suspensions such as whole blood, it is, therefore, essential to understand how the trajectories of particles moving through a DLD array are affected — not only by the device geometry, but also by the interactions with surrounding RBCs and consequently by the hematocrit of the suspension.

Here, we perform a combined experimental and simulation study to elucidate the physical mechanisms of particle behavior in concentrated erythrocyte suspensions and the effect of hematocrit on the sorting efficiency of DLD devices. In the following, we will use the term RBC suspensions. This is strictly true for the simulations where only normal disc-shaped erythrocytes are considered. However, for the experiments, resuspended blood will be used such that other cell types are also present. Most microfluidic experiments are performed using a single-section device (with a constant row shift) to avoid overlay of different displacement mechanisms. Simulations, which explicitly represent deformable RBCs embedded in a Newtonian fluid, are employed to elucidate the physical mechanisms that govern the behavior of particles at finite hematocrit. We use spherical particles with diameters in the range of $2-10 \mu \mathrm{m}$ as models for cells and microorganisms to facilitate a systematic study of the effect of particle size and hematocrit on sorting capability. Our main result is that sorting remains possible up to surprisingly high hematocrit values. However, the sorting is no longer completely deterministic, because the presence of RBCs leads to an increased dispersion. The dispersion by RBCs has the strongest effect on particles that are close to the critical size of the DLD array. This study provides an understanding of the mechanisms, which govern the dispersion effect of hematocrit on particle sorting, and quantifies the performance of DLD devices for particle isolation from concentrated RBC suspensions.

\section{MATERIALS AND METHODS}

\section{A. Microfluidic devices}

Two devices are used in this work. Device 1 is a chirped array with critical diameters ranging from $D_{C}=2.9$ to $D_{C}=9.0 \mu \mathrm{m}$. Device 2 is a uniform array with one critical size $D_{C}=6.1 \mu \mathrm{m}$. Note that the critical sizes here are estimated using an empirical formula from Ref. [41]. Design details are described in the Supplemental Material [42].

Devices are fabricated from polydimethylsiloxane (PDMS) (Sylgard 184, Dow Corning, Midland, MI, USA) using standard replica molding [43] of SU8 (SU8-2010, Microchem Corp., MA, USA) structures on a $3^{\prime \prime}$ silicon substrate. Designs are drawn in L-Edit 11.02 (Tanner Research, Monrovia, CA, USA), the photomask is printed by Delta Mask (Delta Mask, Enschede, The Netherlands), and the master is fabricated using UV lithography (Karl Suss MJB4, Munich, Germany). To facilitate demolding from the master, it is coated with an antisticking layer, 1H,1H,2H,2H-perfluorooctyltrichlorosilane (ABCR GmbH \& Co. KG, Karlsruhe, Germany).

PDMS casts are oxygen-plasma bonded to microscope slides spun with a thin layer of PDMS (Plasma Preen II862, Plasmatic Systems Inc., North Brunswick, NJ, USA) to obtain devices with all internal surfaces consisting of PDMS. Immediately after bonding, the devices are filled with a solution of $0.2 \%$ (weight/volume) PLL(20)-g[3.5]PEG(2) (SuSoS AG, Dubendorf, Switzerland) in deionized water and left for $20 \mathrm{~min}$ before subsequent flushing in order to minimize the adhesion of cells to the walls.

Silicon tubing with 3-mm-inner and 5-mm-outer diameters (228-0725 and 228-0707, VWR International LLC, Radnor, PA, USA) are glued (Elastosil A07, Wacker 
Chemie AG, Munich, Germany) onto the device as reservoirs.

\section{B. Measurement setup}

A MFCS-4C pressure controller (Fluigent, Paris, France) is used to control the overpressure at the inlets, and the outlet reservoirs are kept at ambient pressure. Images are captured through an inverted Nikon Eclipse Ti microscope (Nikon Corporation, Tokyo, Japan) with an Andor NEO scientific CMOS camera (Andor Technology, Belfast, Northern Ireland) and Lumencor SOLA light engine ${ }^{\mathrm{TM}}$ (Lumencor Inc, OR, USA) with fluorescein isothiocyanate (FITC), tetramethylrhodamine (TRITC), and 4',6-diamidino-2-phenylindole (DAPI) filters or brightfield. $4 \times$ (Nikon Plan Fluor NA0.13), $10 \times$ (Nikon Plan Fluor NA0.3), and $60 \times$ (Nikon Fluor water immersion NA 1.0) objectives are used and all movies are acquired at 10 frames per second.

The results for both experiments and simulations are characterized by the displacement index, $I_{S}$, which is defined as the ratio of the average lateral displacement of particles per post to the row shift. For the ideal displacement mode, $I_{S}=1$ as the particles are displaced laterally from their original stream and flow along the gradient of the post array. For the ideal zigzag mode, $I_{S}=0$ as the particles move in the direction of the flow without net lateral displacement.

\section{Reagents and blood samples}

Whole blood is collected by finger pricking from healthy volunteers and mixed 1:20 with autoMACS ${ }^{\mathrm{TM}}$ running buffer (EDTA, BSA, Na-azide in $1 \times$ phosphate buffered saline). The resulting suspension is then spun down to achieve a concentration equivalent to whole blood. Since the vast majority of the cells in the suspension are erythrocytes, we use the term RBC suspension throughput the text.

For device 1, fluorescent polystyrene particles (4.9 \pm $0.3 \mu \mathrm{m}$, Polysciences Inc, USA) at a concentration of $0.01 \%$ (volume/volume) are injected into the particle inlet of the device. The measurements are conducted in the RBC suspension at different concentrations, $c_{0}=0, c_{1}=10^{6} \mu \mathrm{L}^{-1}, c_{2}=2 \times 10^{6} \mu \mathrm{L}^{-1}$, and $c_{3}=4.5 \times 10^{6} \mu \mathrm{L}^{-1}$, corresponding to approximately $0 \%$, $10 \%, 20 \%$, and $45 \%$ hematocrit.

For device 2, experiments are performed by flowing suspensions of polystyrene microspheres of diameters $1.8 \pm 0.1$ and $4.7 \pm 0.2 \mu \mathrm{m}$ (no. 17687 , no. 18340 , Polysciences Inc.), $6.5 \pm 0.6$ and $9.8 \pm 0.7 \mu \mathrm{m}$ (no. 35-2, no. 36-3, Duke Scientific Corp.) and $7.8 \pm 0.1 \mu \mathrm{m}$ (PSFluoGreen-Fi220, microParticles $\mathrm{GmbH}$ ), while simultaneously flowing solutions of RBC suspensions into the background inlets at hematocrits of $0 \%, 5 \%, 15 \%, 30 \%$, and $45 \%$. The lateral positions (degree of displacement) of the microspheres are measured at the end of the separation array and a displacement index $(0=$ ideal zigzag mode and $1=$ ideal displacement mode) is calculated.

For each experiment, each inlet is fed with suspensions of identical RBC concentrations that are quantified at the beginning of the DLD device.

Automated scripts are used to analyze the exit distribution of the sorted particles. The script is based on an analysis of intensity of the fluorescent particles after compensation for background. Each data point is derived from more than 100 particles. Due to the larger concentration in terms of number or particles per unit volume, the smaller particles are sampled to a greater extent than the larger particles.

\section{Simulations}

Simulations take place with design parameters corresponding to device 2 (see the Supplemental Material [42]). To model fluid flow within a microfluidic device, we employ the dissipative particle dynamics (DPD) method $[44,45]$, which is a mesoscopic hydrodynamics simulation technique. In this method, the simulated system consists of a collection of $N$ particles with mass $m_{i}$, position $r_{i}$, and velocity $v_{i}$, and each individual particle represents a cluster of atoms or molecules. The dynamics of the DPD particles is governed by Newton's second law of motion, and the total force acting between two particles within a selected cut-off $\left(r_{C}\right)$ region is a sum of pairwise conservative, dissipative, and random forces.

Modeling and simulation of blood flow on the cellular level have made enormous progress in recent years [46]. Here, we employ two-dimensional (2D) simulations to study the effect of the hematocrit on the separation of rigid spherical particles in DLD devices with circular post arrays. Both RBCs and rigid spherical particles are modeled as closed bead-spring chains with $N_{V}$ particles ( $N_{V}=50$ for RBCs and $N_{V}=30$ to 60 for rigid spherical particles depending on their size) connected by $N_{S}=N_{V}$ springs, as illustrated in Fig. 1(b). The bonding potential, bending energy, and area constraint are applied to control the shape and rigidity of the cells and particles [15]. These models have been validated to be able to capture the essential physical features required to correctly describe the motion of particles in conventional DLD devices with circular posts [15].

The simulated domain, which contains four posts, is periodic in both directions [15]. We verify that four posts are enough to avoid periodicity effects by running a limited set of simulations on an even larger domain. Boundary conditions in the $x$ direction (the flow direction) are subject to a constant shift $\Delta \lambda$ in the $y$ direction in order to mimic the shift between two consecutive rows of posts. Therefore, a shift is introduced for every boundary crossing event. The flow in the $x$ direction is driven by a force applied to each 
(a)

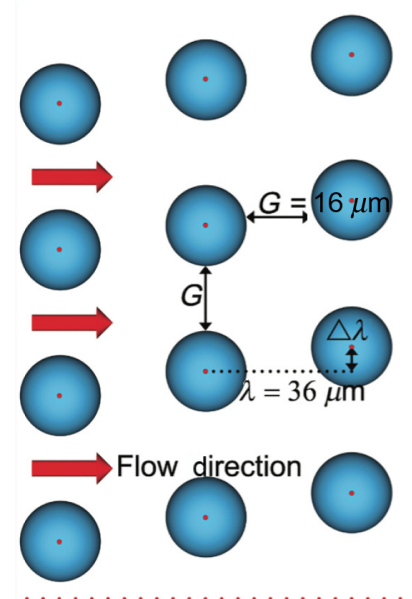

(b)

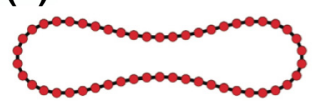

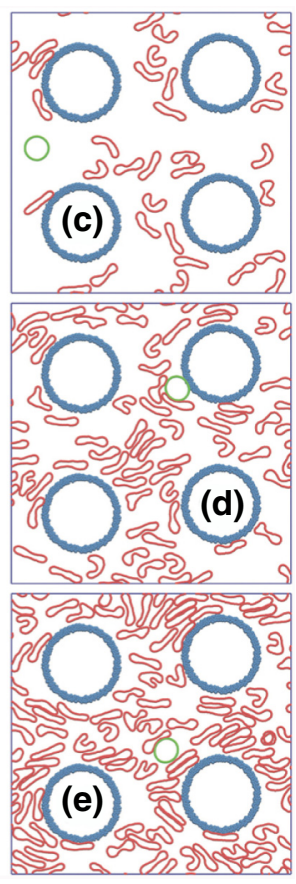

FIG. 1. Schematic illustrations of the simulated DLD system. (a) DLD array with circular posts. The geometry is defined by the post center-to-center distance $\lambda$, the post gap $G$, and the row shift $\Delta \lambda$. (b) 2D models of RBCs and rigid spherical particles. (c)-(e) Instantaneous snapshots of typical fluid structures at different hematocrits: (c) $11.3 \%$, (d) $22.6 \%$, and (e) $33.9 \%$. DLD device parameters are $\lambda=36, \Delta \lambda=2.4$, and $G=16 \mu \mathrm{m}$ corresponding to device 2 (see the Supplemental Material [42]).

fluid particle in order to control the flow rate. To enforce no net flow in the $y$ direction, which occurs in the device due its side walls, a force in the $y$ direction is also applied to each fluid particle. This force is adapted separately for every hematocrit to satisfy the no-net-flow condition.

\section{RESULTS AND DISCUSSION}

\section{A. Proof of principle: WBC enrichment at $45 \%$ hematocrit using a standard DLD device}

To demonstrate the usefulness of DLD sorting at high hematocrit, Figure 2 shows that WBCs can readily be enriched from a concentrated RBC sample using a standard DLD device (device 1, see the Supplemental Material [42]). The DLD device consists of 13 separate sections with $D_{C}$ ranging from approximately 3 to $9 \mu \mathrm{m}$. Full details of the device design can be found in the Supplemental Material [42] and in Ref. [20]. A rough estimate based on cell counting at the end of the device gives us an approximate ratio of one WBC per five RBCs. This means that an enrichment factor of about 200 is achieved in comparison to the physiological ratio of one WBC per 1000 RBCs in whole blood.

We use the same device (device 1, see the Supplemental Material [42]) to study the displacement of polystyrene microspheres. Figure 3 presents outlet distributions of the particles for different hematocrits, indicating that the effective size of the particle decreases as the hematocrit is increased, that is, lateral displacement of particles decreases. At the same time, the width of the distribution increases with increasing hematocrit. These results suggest that sorting must still be possible at high hematocrits, even though RBCs at finite hematocrit lead to significant changes in particle trajectories when compared to those at zero hematocrit.

\section{B. Particle displacement in a single-section DLD device with a constant row shift}

In order to better understand the effect of RBCs on the trajectories of suspended particles, a different device is built with a carefully selected row shift $\Delta \lambda=2.4 \mu \mathrm{m}$ in

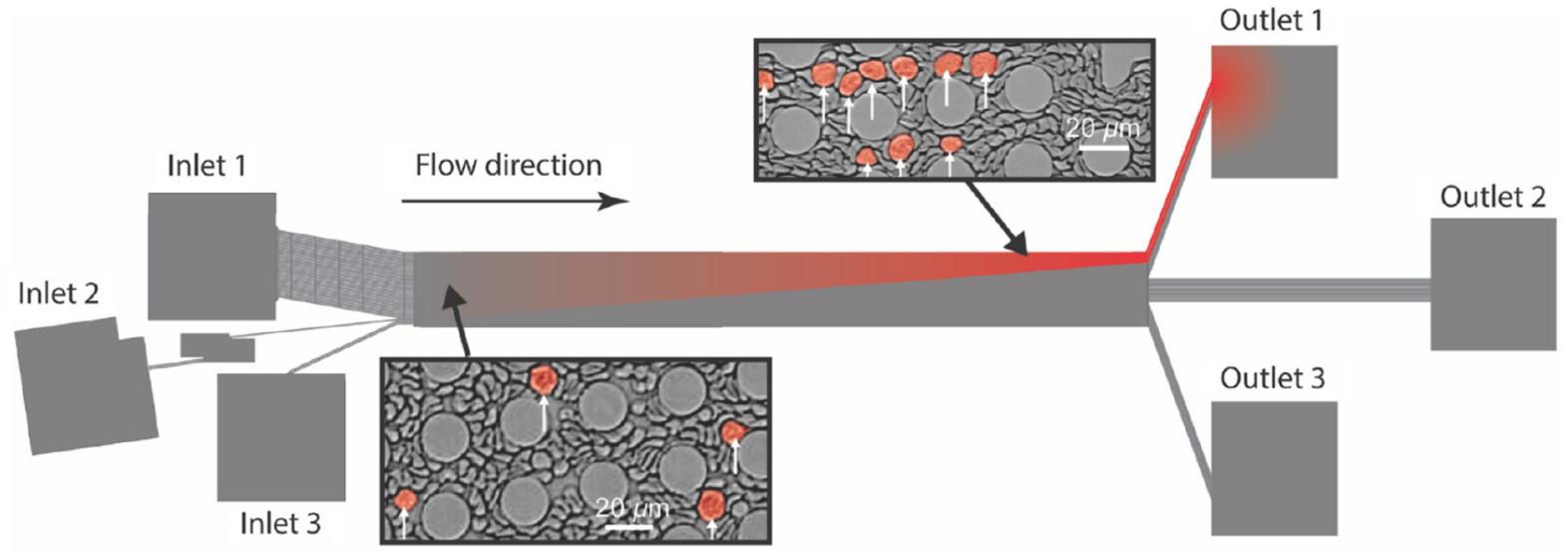

FIG. 2. Enrichment of WBCs at 45\% hematocrit using a DLD device (device 1, see the Supplemental Material [42]) with 13 separate sections, corresponding to $D_{C}$ ranging from approximately 3 to $9 \mu \mathrm{m}$ in steps of $0.5 \mu \mathrm{m}$ [20]. The device is run with an overpressure of $100 \mathrm{mBar}$ at the inlets. Left image shows an elevated concentration of WBCs in the middle of the device and the right image shows an even larger concentration at the end. WBCs are indicated by white arrows. Corresponding videos are available in the Supplemental Material [42] (videos 1a and 1b). 
Theoretical $D_{c}(\mu \mathrm{m})$

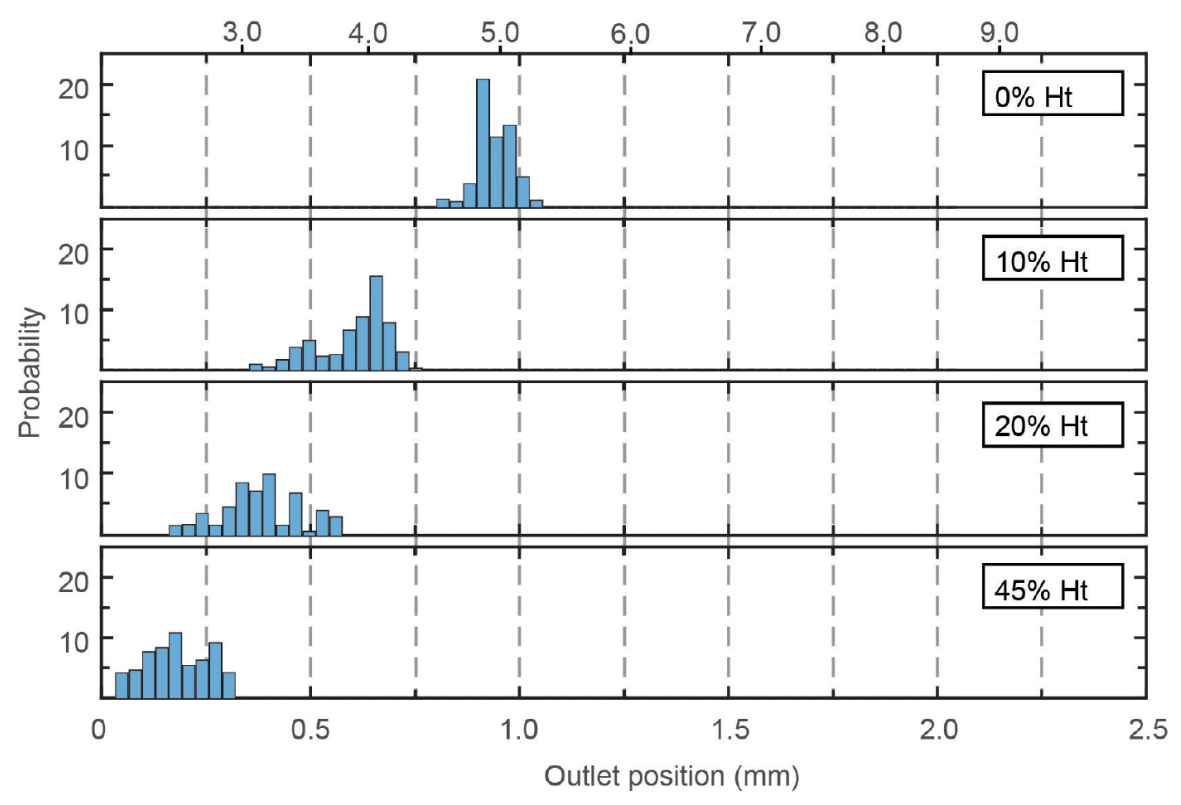

FIG. 3. Experimental exit distributions of polystyrene particles (diameter $4.9 \pm 0.3 \mu \mathrm{m}$ ) using the DLD device with multiple sections (device 1, see the Supplemental Material [42]) for various hematocrits. The device is run with an overpressure of $100 \mathrm{mBar}$ at the inlets. The higher the hematocrit, the smaller the effective size of the polystyrene beads. On the top, the corresponding critical sizes from an empirical formula in Ref. [41] are given for the different outlet positions. This provides a scale for the effective sizes of the sorted particles. a single section to avoid the overlay of different motions in integrated multisections, Fig. 4 (device 2, see the Supplemental Material [42]). With a sufficiently large critical size, the RBCs inherently move in the zigzag mode, and therefore, exhibit a homogeneous cell distribution throughout the device. A systematic study can now be performed to examine the sorting performance for a variety of particle sizes and hematocrits.

As a reference, we perform standard DLD separation of microspheres of various sizes (see the Supplemental Material [42] for characterization of microsphere sizes) in an aqueous buffer solution without RBCs, that is, $0 \%$ hematocrit. Figure 5(a) shows that spheres with diameters of 1.8 and $4.7 \mu \mathrm{m}$ closely follow the average fluid flow direction unperturbed by the effects of the DLD array (ideal zigzag mode). In contrast to the small particles, spheres with diameters of 7.8 and $9.8 \mu \mathrm{m}$ travel through the entire device in the displacement mode. Spheres with a diameter of $6.5 \mu \mathrm{m}$ have a mean displacement index of 0.4 , even though their diameter is slightly larger than the theoretical $D_{C}=6.1 \mu \mathrm{m}$. Interestingly, their trajectories have a much broader distribution than the other microspheres. This behavior corresponds to a so-called mixed mode, which has been observed both in experiments and simulations [14-17], and is due to broken flow symmetry [16] and anisotropic permeability $[14,17]$ in DLD devices. In addition, two technical issues might be important here when the size of the beads is close to the critical size of the devices. First, the size distribution of the beads spans the range below and above the critical size and second, the effect of any defects in the DLD array is amplified.

From the experiments where the microsphere particles are mixed in RBC suspensions [Fig. 5(a)], four different regimes can be identified [representative videos are available in the Supplemental Material [42] (video 2)]. To clearly see the effect of the RBCs, we consider both the indicated averages as well as the range of values delimited by the error bars.

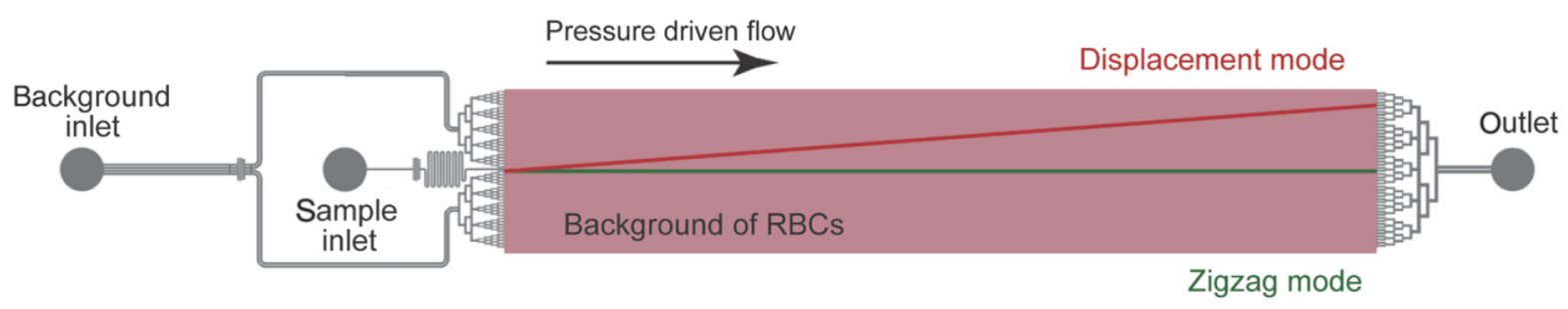

FIG. 4. DLD device with a single section having a constant row shift (device 2, see the Supplemental Material [42]). Post diameter $20 \mu \mathrm{m}$, height $15 \mu \mathrm{m}$, gap size $G=16 \mu \mathrm{m}$, periodicity $N=15$, corresponding to a row periodicity length $\lambda=36 \mu \mathrm{m}$ and a row shift $\Delta \lambda=2.4 \mu \mathrm{m}$, giving us a nominal $D_{C}=6.1 \mu \mathrm{m}$ based on Ref. [41]. The device has two inlets: one central inlet for the microspheres and one inlet for the RBC suspension. The latter is guided to two wide flow streams on either side of the particle flow stream. 

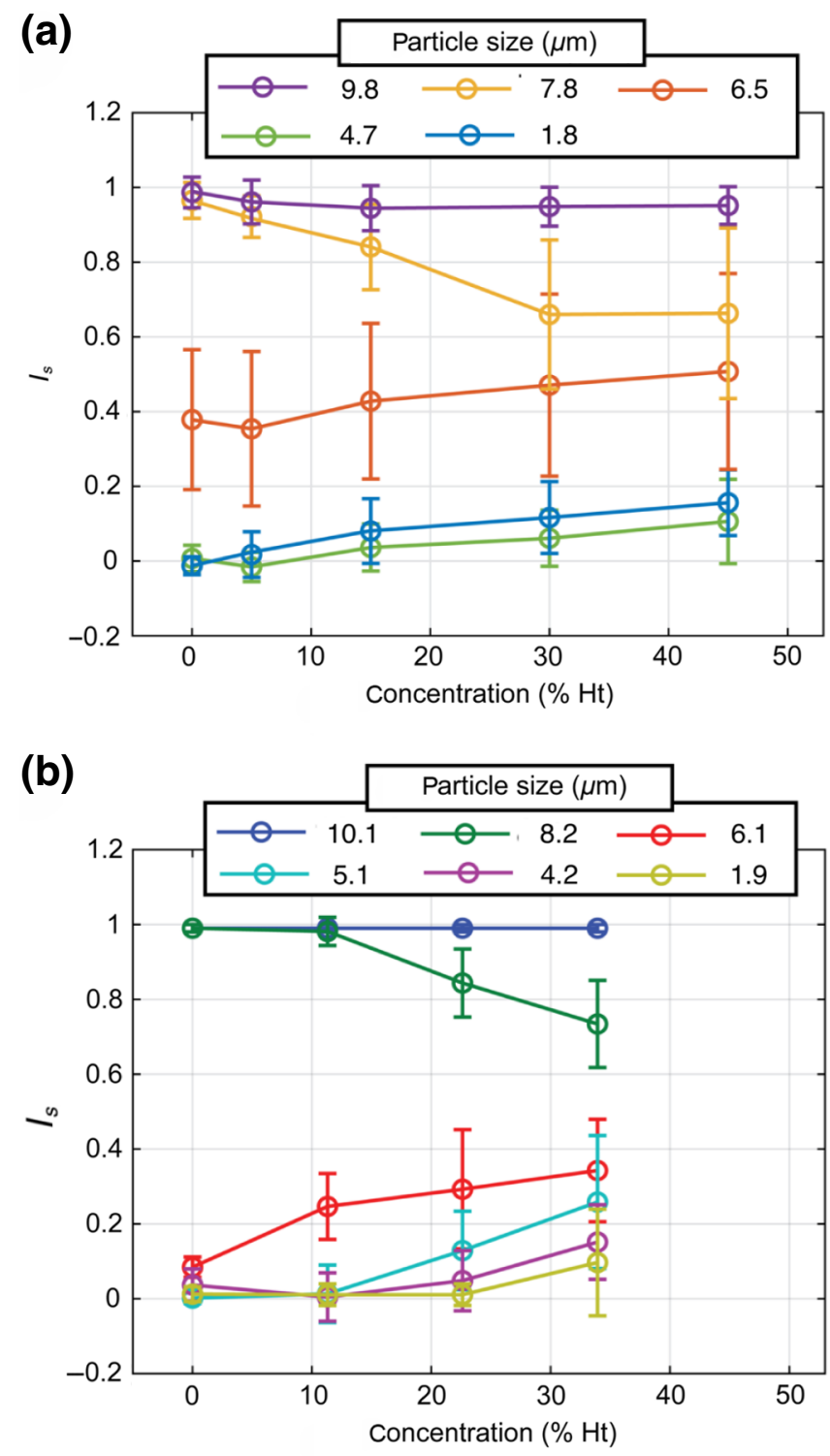

FIG. 5. Effect of hematocrit on the displacement of microspheres (device 2 with $D_{C}=6.1 \mu \mathrm{m}$, see the Supplemental Material [42]). (a) The displacement index as a function of hematocrit obtained from the experiments at a 100-mBar driving pressure for five different microsphere diameters, $D_{P}$. (b) The displacement index from 2D simulations as a function of hematocrit $\left(H_{t}\right)$ for six different microsphere diameters, $D_{P}$.

(a) For $D_{P} \gg D_{C}$, no effect of the RBC background is observed, since particles with $D_{P}=9.8 \mu \mathrm{m}$ consistently exhibit the displacement mode characterized by the displacement index of 1.0 .

(b) When $D_{P}$ is close to and slightly above $D_{C}$, the displacement index decreases as the hematocrit is increased, which is illustrated by the data for $D_{P}=7.8 \mu \mathrm{m}$.

(c) When $D_{P}$ is close to and slightly below $D_{C}$, the increase of hematocrit results in an increase of the displacement index, as seen for $D_{P}=6.5 \mu \mathrm{m}$. (d) For $D_{P} \ll D_{C}$, the displacement index of particles is nearly unaffected by the presence of RBCs. However, there is a slight increase in displacement index and the outlet distribution is wider with increasing hematocrit, as seen for $D_{P}=1.8$ and $4.7 \mu \mathrm{m}$.

Clearly, the effect of hematocrit is strongest for particles whose size is close to the critical size of the device. It is intuitive to expect that suspended particles experience some degree of dispersion due to interactions or "collisions" with flowing RBCs. Higher hematocrits induce more collisions between the RBCs and particles, which increasingly disturb the lateral displacement of the particles and, in turn, affect the separation efficiency. This proposition is also consistent with the observation in Fig. 3 that the outlet distribution broadens with increasing hematocrit.

\section{Underlying mechanisms}

In order to elucidate the mechanisms of how hematocrit affects particle trajectories in DLD devices, simulations of corresponding 2D systems (see Sec. II) are performed to provide a direct and qualitative understanding. We can roughly estimate the critical size from our simulations to be $D_{C}=7.0 \pm 0.5 \mu \mathrm{m}$. The value is slightly larger than the theoretical critical size $D_{C}=6.1 \mu \mathrm{m}$, indicating that the empirical formula gives an approximate value $[16,17]$.

Simulation results for the displacement index as a function of hematocrit for various particle sizes are shown in Fig. 5(b). The simulations are consistent with the trends observed in the experiments in Fig. 5(a). For representative examples from the simulations, see videos in the Supplemental Material [42] (videos 3 and 4).

The following mechanisms are found to be at play.

(1) Shear-flow-induced collisions and RBC pressure: The local gradients in flow velocity result in frequent collisions among RBCs and between RBCs and particles, which lead to an effective pressure proportional to $H_{t}, \dot{\gamma}$, and $\eta_{\text {eff, }}$ where $\dot{\gamma}$ is the local shear rate and $\eta_{\text {eff }}$ is the effective viscosity of the suspension [47]. In homogeneous channels, this pressure reduces the thickness of the RBCfree layer at the channel wall. For our DLD arrays, this pressure has several implications. It increases with increasing hematocrit, generates a more homogeneous distribution of particles in the available volume [Figs. 6(a)-6(c)], and pushes RBCs closer to confining surfaces (Fig. 7). Shear-induced collisions also affect particle margination or migration toward the walls [48].

(2) Layering of RBCs at post surfaces: An immediate consequence of the shear-flow-induced RBC pressure is the formation of a RBC-rich layer at confining surfaces. In addition, in DLD arrays, the flow is not always tangential to the post surface, but in some locations has a strong perpendicular component. This enhances the layering effect 
(a)

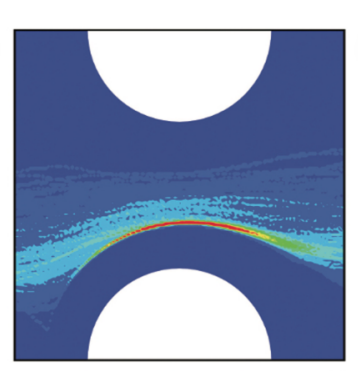

(b)

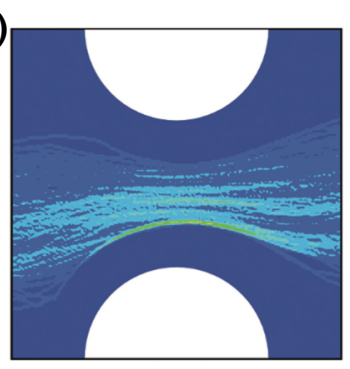

(c)

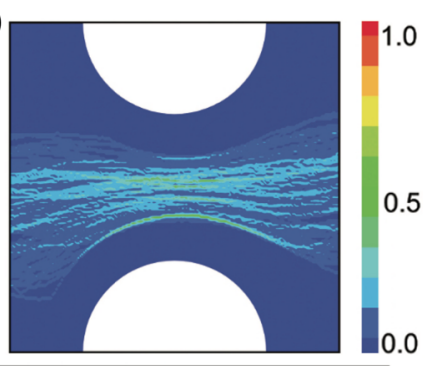

(d)

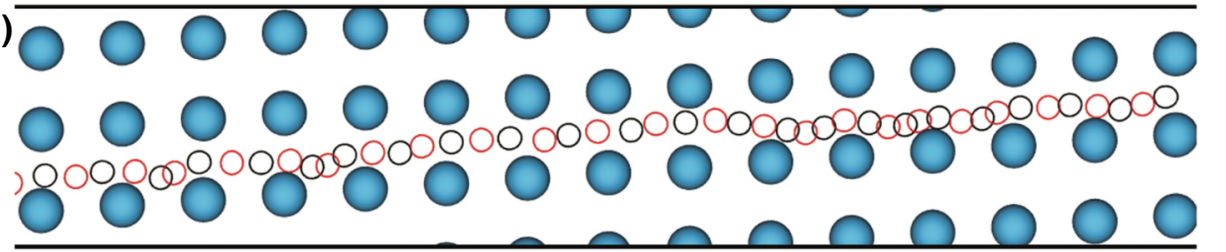

(e)

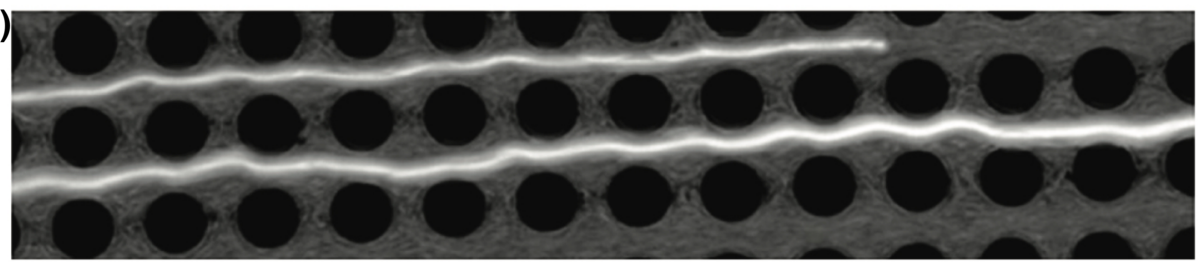

FIG. 6. Bumpy displacement trajectories (simulation and experimental device 2 with $D_{C}=6.1 \mu \mathrm{m}$, see the Supplemental Material [42]) of large particles $\left(D_{P}>D_{C}\right)$ in the presence of RBCs. (a)-(c) Simulated distributions of the suspended particles (center of mass) between two posts for $H_{t}=11.3 \%, \quad H_{t}=22.6 \%$, and $H_{t}=33.9 \%$, respectively $\left(D_{P}=10.1 \mu \mathrm{m}\right)$. (d) Simulated snapshot of a typical bumpy trajectory of the particle with $D_{P}=10.1 \mu \mathrm{m}$ at $H_{t}=22.6 \%$. (e) Experimental trajectories of fluorescent microspheres with a diameter $D_{P}=9.8 \mu \mathrm{m}$ at $30 \%$ hematocrit. in incident flow regions. The distributions of RBCs for various hematocrits are shown in Fig. 7 and clearly demonstrate this effect. However, note that the layering effect should not be interpreted directly as a complete coverage of the posts by RBCs, but refers to inhomogeneous RBC distribution as well as to the parallel orientation of RBCs at the post surface. Inhomogeneity in the RBC distribution is also observed in experiments, but it is difficult to quantify the density distribution.

(3) Go with RBCs: An individual RBC at low hematocrit moves in a zigzag mode through the device. Particles collide with the RBCs frequently, and are thereby dragged into similar trajectories (Fig. 8). With increasing hematocrit, RBCs also attain an increasing displacement component (Fig. 9), which is related to the more homogeneous $\mathrm{RBC}$ distribution in the DLD device.
The consequences of these mechanisms on the four different particle-size regimes are discussed further below.

(a) $D_{P} \gg D_{C}$.

For large particles, both experiments and simulations in Fig. 5 show that RBCs have no effect on the particle trajectories. Obviously, such large particles follow the displacement mode and any additional dispersion due to interactions with RBCs does not alter the displacement mode. Figures 6(d) and 6(e) illustrate typical trajectories of large particles from simulations and experiments at moderate hematocrits. An interesting observation from these trajectories is that the particle is often displaced away from the post as though it bumps off the post. This bumpy behavior is mediated by RBCs, since in an RBC-free suspension, the
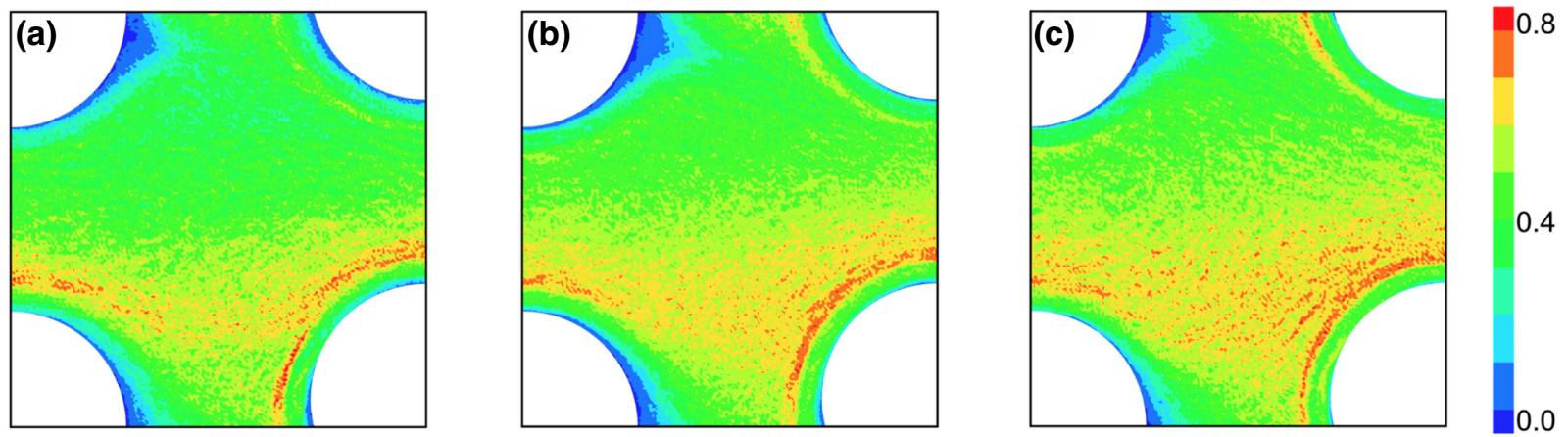

FIG. 7. Simulated distributions of the membrane particles of RBCs at hematocrits of (a) 11.3\%, (b) $22.6 \%$, and (c) $33.9 \%$. Membrane particles represent RBC surfaces, and thus their distribution is similar but not identical to the local hematocrit. The distributions are averaged over several thousands of frames from long simulation trajectories and normalized by the maximum density. 


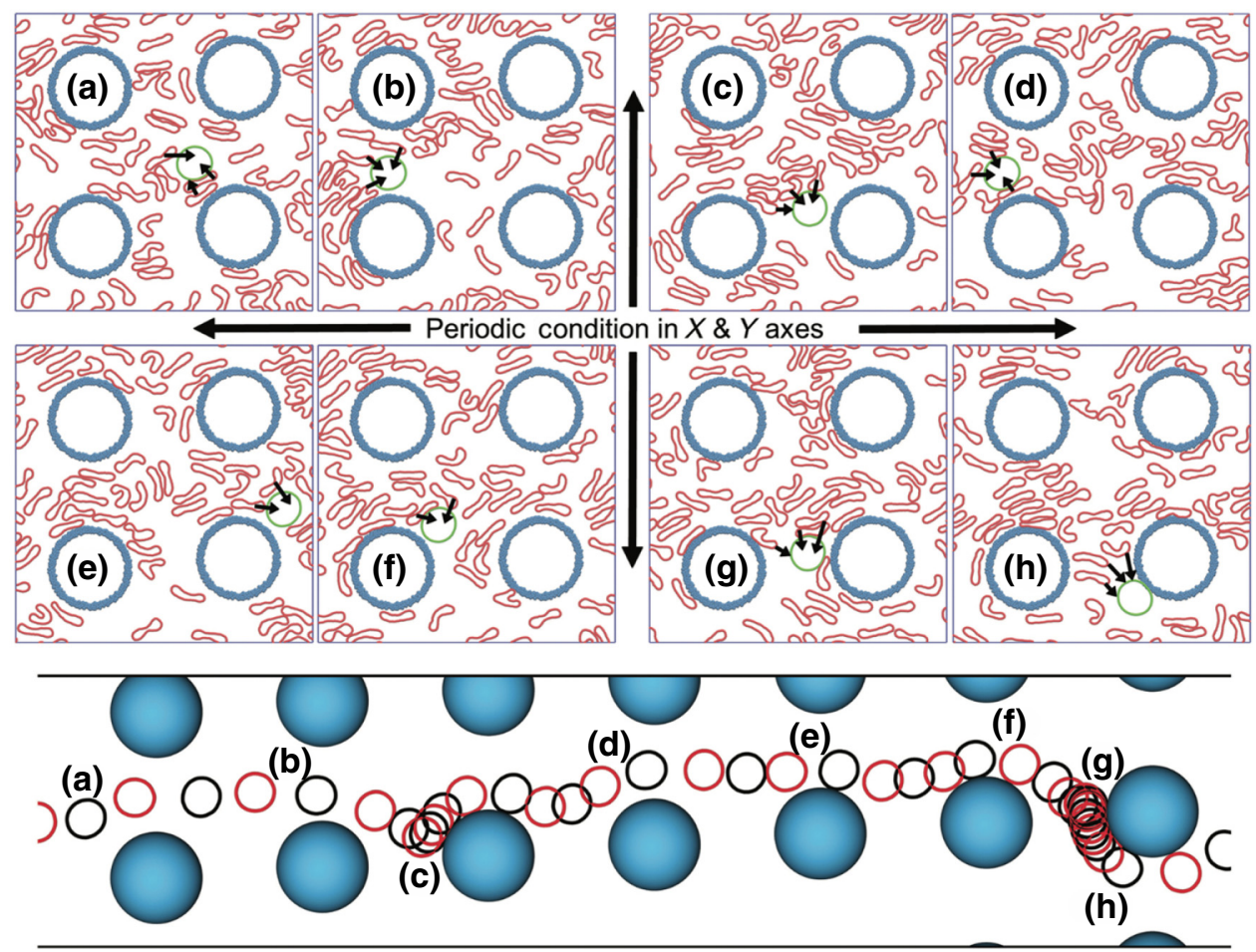

FIG. 8. RBC-induced zigzag motion. Snapshots of typical flow behavior of RBCs and suspended particles (simulation), where a zigzag motion of the particle is promoted due to interactions with RBCs. Here, $D_{P}=8.2 \mu \mathrm{m}>D_{C}$ and $H_{t}=22.6 \%$. Different snapshots are marked by letters $\mathrm{a}-\mathrm{h}$, corresponding to the particle positions in the trajectory displayed in the lower figure.

displacement trajectory of the particle perfectly follows the postarray lane where the particle "touches" every subsequent obstacle. Furthermore, Figs. 6(a)-6(c) further reveal that the distribution of suspended particles gradually shifts away from the posts toward the gap center with increasing hematocrit due to the formation of a high-density RBC layer at the post surface (Fig. 7). This means that the displacement trajectory becomes increasingly bumpy with increasing hematocrit, wherein the particles irregularly

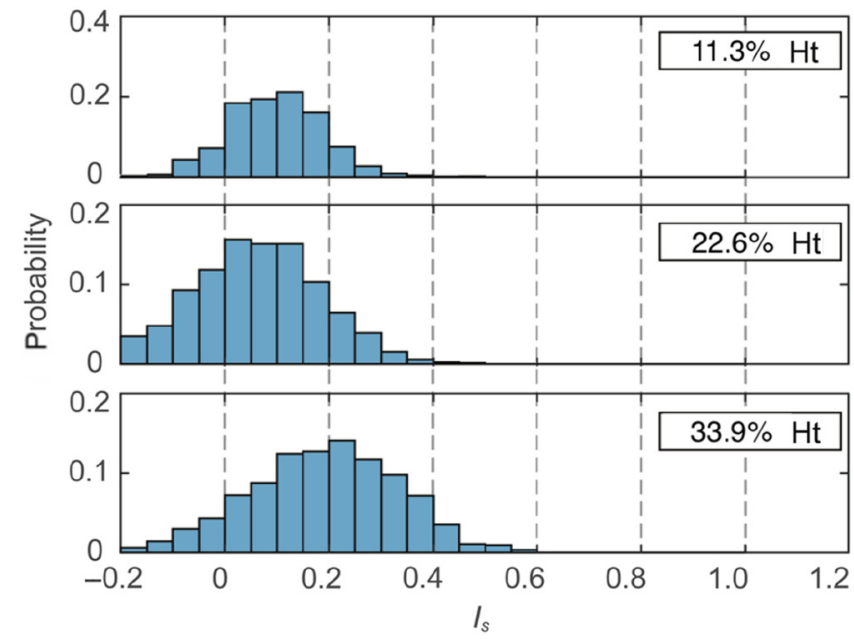

FIG. 9. Simulated outlet distributions of RBCs without any added particles as a function of the displacement index $I_{S}$ at different hematocrits. This shows how hematocrit affects the overall traversal of single RBCs through DLD device 2. move up and down within a lane between posts due to frequent collisions with the RBCs.

(b) $D_{P}$ is close to $D_{C}$, with $D_{P}>D_{C}$.

Particles are expected to exhibit a displacement mode in the buffer solution without RBCs for $D_{P}>D_{C}$. However, at a high enough hematocrit for particles whose size is close to $D_{C}$, the displacement index is found to decrease to be significantly smaller than 1.0, as shown in Fig. 5, implying the appearance of induced zigzag events. Interactions with RBCs increase effective lateral diffusion of the particles, leading to an increased cross-streamline migration. Here, the collisions with RBCs are strong enough to occasionally promote lateral displacement of the particles into zigzagging. Such interactions are illustrated in Fig. 8, where it is apparent that the particle encounters numerous collisions with RBCs. Snapshots in Figs. 8(g) and 8(h) show how several RBCs drive and accompany the particle into the zigzag mode. The "go-with-RBCs" effect obviously depends on the hematocrit, since the zigzagging frequency increases with an increase of hematocrit, which is supported by the reduced displacement index for particles with $D_{P} \approx 8 \mu \mathrm{m}$ in Fig. 5. However, it is clear that the interactions with RBCs favoring a zigzag motion are not strong enough to force the largest particles into zigzagging and they remain in the displacement mode.

Note that the "bumping" effect of RBCs due to their layering near the posts as illustrated in Fig. 7 occurs here as well, which can be seen in Figs. 8(a) and 8(d). Therefore, both "bumping" and "go-with-RBCs" effects 
are present and their interplay influences particle trajectories. For particles whose size is close to $D_{C}$ and $D_{P}>D_{C}$, the "go-with-RBCs" effect is more prominent, resulting in a mixed mode with a displacement index, $0.5<I_{S}<1.0$.

(c) $D_{P}$ is close to $D_{C}$, with $D_{P}<D_{C}$.

Particles naturally assume the zigzag mode without RBCs for $D_{P}<D_{C}$. With increasing hematocrit, the displacement index for such particles increases, as shown in Fig. 5. Again, we need to consider the two effects of RBCs: (i) "layering of RBCs at post surfaces" that favors the displacement mode and (ii) "go-with-RBCs" that promotes zigzagging. Since the displacement index for particles with $D_{P}<D_{C}$ increases, as the hematocrit is elevated, the frequency of zigzagging events must decrease. This indicates that the "bumping" effect is dominant here, resulting in a decreased frequency of lane swapping events with increasing hematocrit.

(d) $D_{P} \ll D_{C}$.

For very small particles, the trajectories remain nearly unaffected by RBCs and follow the neutral zigzag mode. This indicates that the "bumping" and "go-with-RBCs" effects are approximately balanced. The displacement index for small particles in Fig. 5(a) has a slight increasing tendency as the hematocrit is elevated, indicating that the "bumping" effect may modestly decrease the frequency of zigzagging.

\section{Outflow distributions}

Average outflow positions of the particles can be easily estimated from the displacement index in Fig. 5 as they characterize the average particle displacement per one row of posts. To examine the effect of hematocrit on the width of outlet distributions, particle positions at the device exit are monitored. Figure 10 shows particle outlet distributions from simulations and experiments for various particle sizes. Simulated distributions for $D_{P}=6.1 \mu \mathrm{m}$ are qualitatively similar to experimental results for $D_{P}=4.7 \mu \mathrm{m}$. In these cases, the outlet distributions are quite narrow in the absence of RBCs, corresponding to a nearly deterministic zigzag motion. With increasing hematocrit, both simulated $\left(D_{P}=6.1 \mu \mathrm{m}\right)$ and experimental $\left(D_{P}=4.7 \mu \mathrm{m}\right)$ distributions shift to a larger average particle displacement and significantly widen since RBCs introduce dispersion to particle trajectories. Therefore, RBCs destroy the strictly deterministic nature of particle trajectories observed in a buffer solution. The dispersion effect of RBCs may also be nonmonotonic as the distribution for $H_{t}=33.9 \%$ from simulations appears to be slightly narrower than that for $H_{t}=22.6 \%$. Clearly, the width of outlet distributions depends on the length of a device or the total number of rows, since the dispersion has a cumulative effect. Thus, outlet distributions are expected to widen when the length of a DLD device is increased. Figure 10 also presents experimental particle outlet distributions for $D_{P}=6.5$ and $D_{P}=7.8 \mu \mathrm{m}$. In the case of $D_{P}=7.8 \mu \mathrm{m}$ with increasing hematocrit, the average particle displacement within the device slightly decreases, which is consistent with a decrease in the displacement index in Fig. 5(a), and the outlet distributions substantially widen. The outlet distributions for experiments with $D_{P}=6.5 \mu \mathrm{m}$ are very broad for all hematocrits (even without RBCs), because the particle diameter is very close to the critical size of the device. Thus, the presence of RBCs leads to significant widening of outlet distributions with the strongest effect for particles whose diameters are close to the DLD critical size.

It is also interesting to take a look at the trajectories of each single RBC. Figure 9 shows outlet distributions for RBCs at different hematocrit values. The width of RBC distributions increases with increasing hematocrit, which is consistent with an increase in the width of outlet distributions for suspended particles. Furthermore, there is a tendency for the RBCs to increase the displacement index as hematocrit is elevated, which can be seen from a shift of the distributions toward more positive values in Fig. 9. The effective size of RBCs is smaller than $D_{C}$, so that the slight increase of the displacement index with increasing hematocrit is consistent with the analogous effect for small particles $\left(D_{P} \ll D_{C}\right)$ in Fig. 5. A nonzero displacement index for RBCs results in their slow positive migration within the DLD device. Thus, for a sufficiently long device, the distribution of RBCs far enough from the inlet may not be uniform with a varying local hematocrit across the device cross section.

\section{E. Other potential contributions affecting particle trajectories}

The "bumping" and "go-with-RBCs" effects of RBCs discussed above provide an intuitive explanation for the behavior of suspended microspheres in DLD devices. However, this clearly does not exclude the possibility that other contributions affecting particle trajectories might be present. For instance, particle margination or migration toward the walls of the device $[9,49]$ may contribute to the lateral motion of the particles. Due to the low lateral speed of particle margination, it is typically demonstrated in long channels. For example, Hou et al. used 5-mm-long channels [10]. For particles moving between posts, there is no obvious net buildup of lateral translation, making their margination not very probable. Furthermore, the particles are partially repelled from the posts due to an increased local hematocrit around the posts (i.e., the "bumping" effect, Fig. 7). Finally, interactions or collisions of microspheres with RBCs also represent one of the margination 
mechanisms, often referred to as shear-induced diffusion [48]. Therefore, it is not possible to make a clear separation between possible margination contribution and the suggested "bumping" and "go-with-RBCs" effects.

Another possible contribution, which may affect particle trajectories in DLD devices, is altered velocity profile due to an increase of the hematocrit. An altered velocity profile in the DLD device can consequently change the critical size of the device. Our simulations show that a change in the average velocity profile is very small for all hematocrits studied, indicating that this effect on the critical size can practically be neglected. This is likely due to the two reasons: (i) we investigate particle motion at moderate hematocrits up to about $33 \%$ and (ii) the DLD structure naturally leads to a continuous mixing, preventing the formation of regions with very high hematocrit values. The latter means that the RBC suspension within the DLD device does not have strong variations in local viscosity, and thus no significant changes in the velocity profile should be expected. (a)

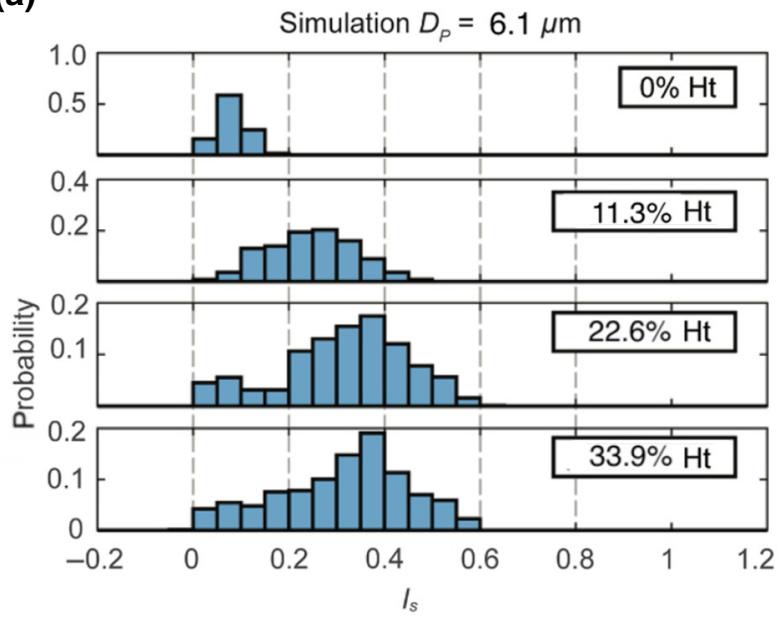

(c)

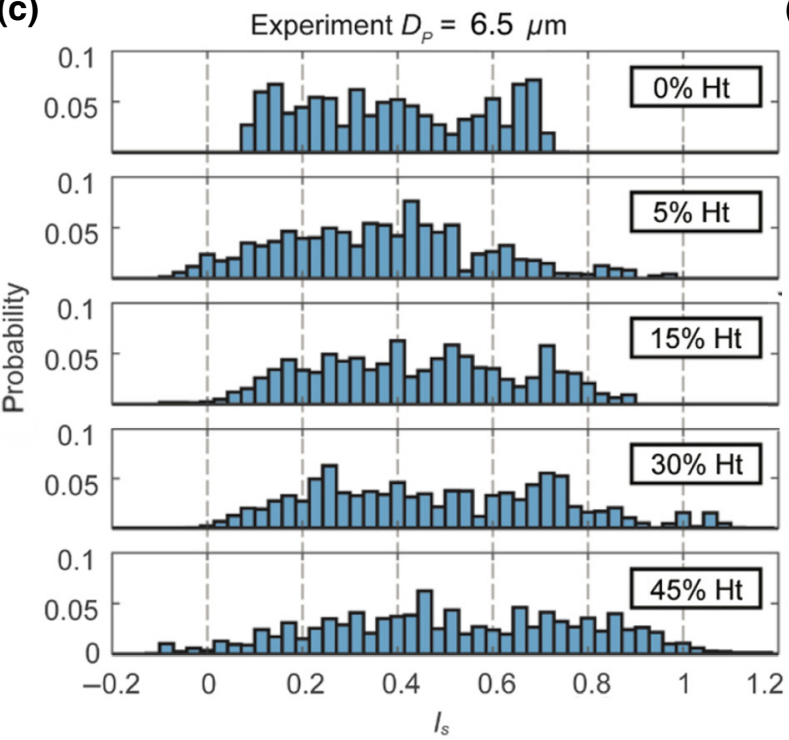

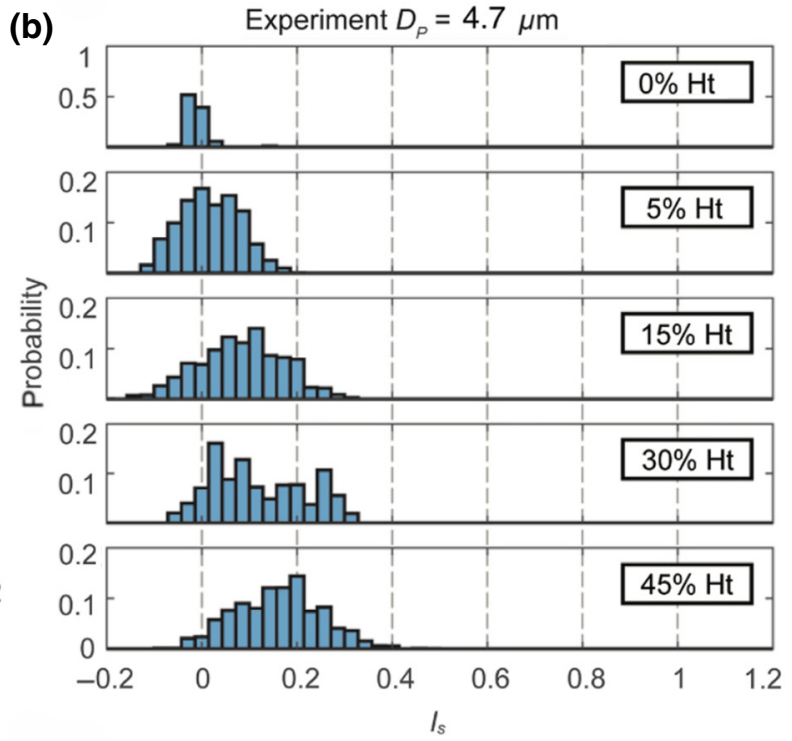

(d)

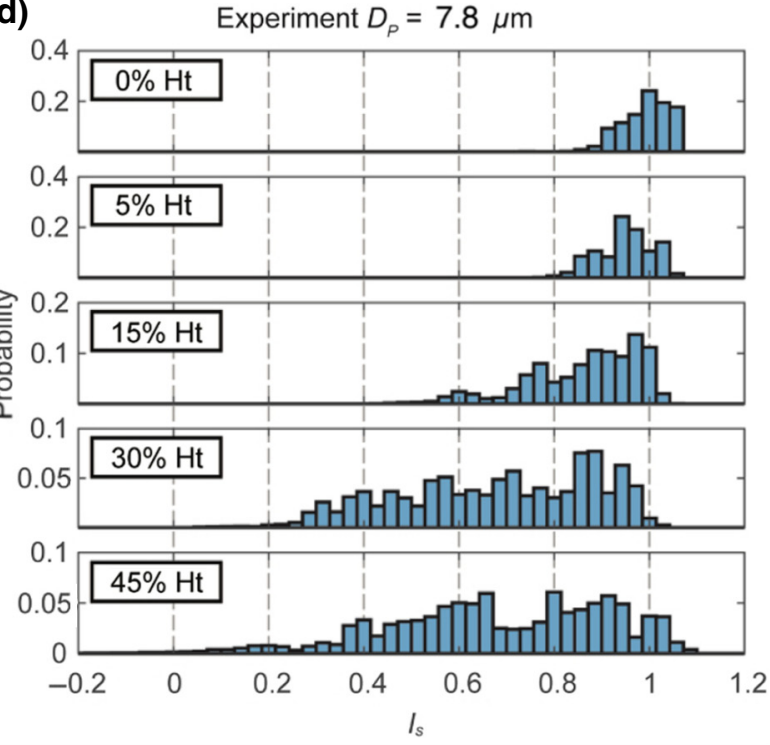

FIG. 10. Outlet distributions from simulations and experiments for different particle diameters as a function of the displacement index $I_{S}$. (a) Simulation results for $D_{P}=6.1 \mu \mathrm{m}$ and (b) experiments for $D_{P}=4.7 \mu \mathrm{m}$ compare well qualitatively and both exhibit an increase in the displacement with increasing hematocrit. (d) Experimental data for $D_{P}=7.8 \mu \mathrm{m}$ shows a decrease in the particle displacement as hematocrit is elevated. In all cases, outlet distributions generally widen with increasing hematocrit. (c) The outlet distributions for experiments with $D_{P}=6.5 \mu \mathrm{m}$ are very broad for all hematocrits (even without RBCs) because the particle diameter is very close to the critical size of the device. 


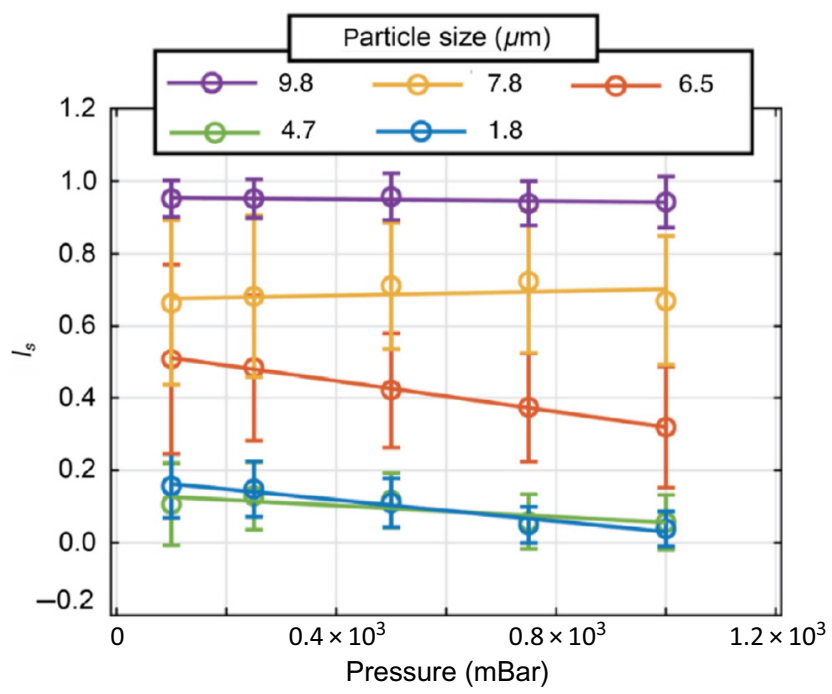

FIG. 11. The effect of flow rate (applied pressure drop) on the displacement index at $45 \%$ hematocrit for various particle diameters. Experimental results (device 2 with $D_{C}=6.1 \mu \mathrm{m}$, see the Supplemental Material [42]).

\section{F. Effect of flow rate}

Flow rate through a DLD device is an important parameter since it is directly related to the throughput of the device. Figure 11 shows the effect of flow rate or the applied pressure drop on the displacement index of various particles at $45 \%$ hematocrit. For particles with $D_{P}>D_{C}$, no significant influence of flow rate on the displacement index is found. This indicates that the "go-with-RBCs" effect of RBCs, which promotes a zigzagging motion, remains unaffected by the flow rate. For particles with $D_{P}>D_{C}$, there is a tendency of decreasing displacement index with increasing the pressure drop, indicating that the "bumping" effect of RBCs, which favors the displacement mode, is reduced. This is likely to occur due to RBC deformation within the DLD device, such that a thinner layer of $\mathrm{RBCs}$ near the posts is formed at high flow rates. In conclusion, the effect of flow rate on the displacement index of suspended particles appears to be quite moderate for a wide range of particle sizes, permitting the use of high pressure drops without strong changes in the efficiency of particle sorting or isolation from concentrated RBC suspensions. In fact, as the flow rate is increased, some of the reduced separation efficiency due to the high hematocrit is regained, indicating that high throughput separations with good resolution might be easier to achieve by combining high hematocrit with high flow rates.

\section{SUMMARY AND CONCLUSIONS}

Our results show that particles within an RBC suspension encounter numerous collisions with the crowding RBCs, which can be roughly divided into two classes on the basis of the corresponding effect on the particle's movement. The first one is a "bumping" effect due to an increased local hematocrit around the posts (Fig. 7), which hinders the particle from entering the lower neighboring streams and favors the displacement motion, as illustrated in Fig. 6. The second effect is denoted as "go-with-RBCs," which forces the particle to flow into the lower neighboring streams and promotes the zigzag motion, as shown in Fig. 8. Therefore, the motion of particles in concentrated RBC suspensions is determined by the competition between these two effects and is generally not fully deterministic anymore.

The influence of these two contributions of RBCs on particle trajectories strongly depends on the ratio between particle size and the critical size of the device. For large enough particles $D_{P} \gg D_{C}$, both effects are not able to deteriorate the stable displacement mode of the particles, resulting essentially in no effect of hematocrit on particle trajectory. In fact, this is perfectly consistent with the successful separation of large cells from whole blood, such as WBCs [34,35] and CTCs [36-39]. When the particle size is close to $D_{C}$ and $D_{P}>D_{C}$, the "go-with-RBCs" effect is able to occasionally force the particles into a zigzag motion such that the particles switch from the pure displacement mode to a mixed mode as the hematocrit is elevated. If the particle size is close to $D_{C}$ and $D_{P}<D_{C}$, the "bumping" effect of RBCs provides a dominant contribution and the particles exhibit an increase in their displacement index with increasing hematocrit. Finally, for very small particles with $D_{P} \ll D_{C}$, there is nearly no effect of hematocrit with a slight tendency of increasing displacement index at higher hematocrits. Clearly, the closer the diameter of the particles to the critical size of a DLD device, the larger the effect of RBCs is. In this range of particle diameters, RBCs are able to significantly perturb or disperse the particle trajectory, which would be deterministic without RBCs.

It is also important to discuss the limitations of the current investigation. Even though concentrated RBC suspensions with hematocrits similar to whole blood are studied, they do not represent the full complexity of whole blood. For example, RBCs in blood are subject to aggregation interactions, which are most prominent at low shear rates or in stasis [50,51]. Such interactions need to be considered in future studies. However, they likely play a negligible role in high-throughput microfluidics with large flow and shear rates, because aggregates are torn apart by high shear forces. The main limitation of the employed simulations is that they are performed in two dimensions. Clearly, 2D simulations cannot capture the full complexity of RBC deformation and hydrodynamic interactions in three dimensions. However, from a qualitative perspective, the results are expected to be reliable because (i) the DLD geometry imposes a quasi-2D flow between two planar walls and (ii) RBCs traversing in deep enough devices generally attain an upright orientation (due to flow and 
collisions with the posts), with their midplane cross section corresponding to the 2D model. Thus, while detailed quantitative predictions are not possible, mechanisms are elucidated and semiquantitative results are obtained.

In conclusion, the main result of our study is that the separation of particles at high hematocrits up to $45 \%$ still remains possible, even though the RBCs may significantly bias and disperse the particle trajectories. RBCs primarily influence trajectories of particles whose sizes are close to the critical size of the DLD array, thereby decreasing the resolution offered by a DLD device under these conditions. However, for certain conditions, when the critical size of a device is far enough from the size of the particles of interest, the effect of a high hematocrit is negligible. For example, for the particles with $D_{P}=7.8$ and $D_{P}=9.8 \mu \mathrm{m}$ used in our experiments at high hematocrit, the larger particle is nearly unaffected by RBCs and remains in the displacement mode, while the smaller particle exhibits a mixed mode. Therefore, their separate enrichment remains achievable despite the presence of the RBCs. In summary, the DLD approach remains highly promising even at high hematocrits. The identified physical mechanisms for the behavior of rigid spherical particles in concentrated RBC suspensions as well as an overall understanding of the influence of RBCs on the trajectories of the particles forms a strong basis in the optimization process of microfluidic systems based on DLD with respect to throughput and any needs for dilution of blood samples. However, since the bioparticles of interest in the blood stream are deformable to varying degrees and since whole blood is different from the suspensions of the RBCs that we studied, for a complete and detailed understanding of the effect of hematocrit on bioparticle separation, future research should target the sorting of relevant bioparticles of different sizes in whole blood.

\section{ACKNOWLEDGMENTS}

The experimental work and all device processing were carried out within NanoLund at Lund University. We acknowledge funding from FP7-PEOPLE-2013ITN LAPASO "Label-free particle sorting" (Grant No. 607350), NanoLund and the Swedish Research Council (VR) Grant No. 2015-05426. D.A.F. acknowledges funding by the Alexander von Humboldt Foundation. The authors also gratefully acknowledge the computing time granted through JARA-HPC on the supercomputer JURECA at Forschungszentrum Jülich [52].

Stefan H. Holm and Zunmin Zhang contributed equally to this work.

[1] C. W. Shields, IV, C. D. Reyes, and G. P. Lopez, Microfluidic cell sorting: A review of the advances in the separation of cells from debulking to rare cell isolation, Lab Chip 15, 1230 (2015).

[2] M. Toner and D. Irimia, Blood-on-a-chip, Annu. Rev. Biomed. Eng. 7, 77 (2005).

[3] J. A. Davis, D. W. Inglis, K. J. Morton, D. A. Lawrence, L. R. Huang, S. Y. Chou, J. C. Sturm, and R. H. Austin, Deterministic hydrodynamics: Taking blood apart, Proc. Nat. Acad. Sci. USA 103, 14779 (2006).

[4] M. Antfolk and T. Laurell, Continuous flow microfluidic separation and processing of rare cells and bioparticles found in blood - a review, Anal. Chim. Acta 965, 9 (2017).

[5] A. Urbansky, P. Ohlsson, A. Lenshof, F. Garofalo, S. Scheding, and T. Laurell, Rapid and effective enrichment of mononuclear cells from blood using acoustophoresis, Sci. Rep. 7, 17161 (2017).

[6] S. Karthick and A. K. Sen, Improved Understanding of Acoustophoresis and Development of an Acoustofluidic Device for Blood Plasma Separation, Phys. Rev. Appl. 10, 034037 (2018).

[7] J. Zhang, S. Yan, D. Yuan, G. Alici, N.-T. Nguyen, M. E. Warkiani, and W. Li, Fundamentals and applications of inertial microfluidics: A review, Lab Chip 16, 10 (2016).

[8] H. Amini, W. Lee, and D. Di Carlo, Inertial microfluidic physics, Lab Chip 14, 2739 (2014).

[9] D. A. Fedosov, J. Fornleitner, and G. Gompper, Margination of White Blood Cells in Microcapillary Flow, Phys. Rev. Lett. 108, 028104 (2012).

[10] H. W. Hou, H. Y. Gan, A. A. S. Bhagat, L. D. Li, C. T. Lim, and J. Han, A microfluidics approach towards highthroughput pathogen removal from blood using margination, Biomicrofluidics 6, 024115 (2012).

[11] H. W. Hou, A. A. S. Bhagat, A. G. L. Chong, P. Mao, K. S. W. Tan, J. Han, and C. T. Lim, Deformability based cell margination - a simple microfluidic design for malaria-infected erythrocyte separation, Lab Chip 10, 2605 (2010).

[12] L. R. Huang, E. C. Cox, R. H. Austin, and J. C. Sturm, Continuous particle separation through deterministic lateral displacement, Science 304, 987 (2004).

[13] D. W. Inglis, J. A. Davis, R. H. Austin, and J. C. Sturm, Critical particle size for fractionation by deterministic lateral displacement, Lab Chip 6, 655 (2006).

[14] T. Kulrattanarak, R. G. M. Sman, Y. S. Lubbersen, C. G. P. H. Schroën, H. T. M. Pham, P. M. Sarro, and R. M. Boom, Mixed motion in deterministic ratchets due to anisotropic permeability, J. Colloid Interface Sci. 354, 7 (2011).

[15] Z. Zhang, E. Henry, G. Gompper, and D. A. Fedosov, Behavior of rigid and deformable particles in deterministic lateral displacement devices with different post shapes, J. Chem. Phys. 143, 243145 (2015).

[16] S.-C. Kim, B. H. Wunsch, H. Hu, J. T. Smith, R. H. Austin, and G. Stolovitzky, Broken flow symmetry explains the dynamics of small particles in deterministic lateral displacement arrays, Proc. Natl. Acad. Sci. USA 114, E5034 (2017).

[17] R. Vernekar, T. Krüger, K. Loutherback, K. Morton, and D. W. Inglis, Anisotropic permeability in deterministic lateral displacement arrays, Lab Chip 17, 3318 (2017).

[18] K. K. Zeming, S. Ranjan, and Y. Zhang, Rotational separation of non-spherical bioparticles using I-shaped pillar 
arrays in a microfluidic device, Nat. Commun. 4, 1625 (2013).

[19] S. Ranjan, K. K. Zeming, R. Jureen, D. Fisher, and Y. Zhang, DLD pillar shape design for efficient separation of spherical and non-spherical bioparticles, Lab Chip 14, 4250 (2014).

[20] S. H. Holm, J. P. Beech, M. P. Barrett, and J. O. Tegenfeldt, Separation of parasites from human blood using deterministic lateral displacement, Lab Chip 11, 1326 (2011).

[21] J. P. Beech, B. D. Ho, G. Garriss, V. Oliveira, B. HenriquesNormark, and J. O. Tegenfeldt, Separation of pathogenic bacteria by chain length, Anal. Chim. Acta 1000, 223 (2018).

[22] M. Jiang, K. Budzan, and G. Drazer, Fractionation by shape in deterministic lateral displacement microfluidic devices, Microfluid. Nanofluid. 19, 427 (2015).

[23] J. P. Beech, S. H. Holm, K. Adolfsson, and J. O. Tegenfeldt, Sorting cells by size, shape and deformability, Lab Chip 12, 1048 (2012).

[24] R. Quek, D. V. Le, and K.-H. Chiam, Separation of deformable particles in deterministic lateral displacement devices, Phys. Rev. E 83, 056301 (2011).

[25] T. Krüger, D. Holmes, and P. V. Coveney, Deformabilitybased red blood cell separation in deterministic lateral displacement devices - a simulation study, Biomicrofluidics 8, 054114 (2014).

[26] Z. Zhang, W. Chien, E. Henry, D. A. Fedosov, and G. Gompper, Sharp-edged geometric obstacles in microfluidics promote deformability-based sorting of cells, Phys. Rev. Fluids 4, 024201 (2019).

[27] E. Henry, S. H. Holm, Z. Zhang, J. P. Beech, J. O. Tegenfeldt, D. A. Fedosov, and G. Gompper, Sorting cells by their dynamical properties, Sci. Rep. 6, 34375 (2016).

[28] J. P. Beech, P. Jönsson, and J. O. Tegenfeldt, Tipping the balance of deterministic lateral displacement devices using dielectrophoresis, Lab Chip 9, 2698 (2009).

[29] K. Loutherback, K. S. Chou, J. Newman, J. Puchalla, R. H. Austin, and J. C. Sturm, Improved performance of deterministic lateral displacement arrays with triangular posts, Microfluid. Nanofluid. 9, 1143 (2010).

[30] K. K. Zeming, T. Salafi, C.-H. Chen, and Y. Zhang, Asymmetrical deterministic lateral displacement gaps for dual functions of enhanced separation and throughput of red blood cells, Sci. Rep. 6, 22934 (2016).

[31] S. Feng, A. M. Skelley, A. G. Anwer, G. Liu, and D. W. Inglis, Maximizing particle concentration in deterministic lateral displacement arrays, Biomicrofluidics 11, 024121 (2017).

[32] D. Holmes, G. Whyte, J. Bailey, N. Vergara-Irigaray, A. Ekpenyong, J. Guck, and T. Duke, Separation of blood cells with differing deformability using deterministic lateral displacement, Interface Focus 4, 20140011 (2014).

[33] K. Loutherback, J. D'Silva, L. Liu, A. Wu, R. H. Austin, and J. C. Sturm, Deterministic separation of cancer cells from blood at $10 \mathrm{~mL} / \mathrm{min}$, AIP Adv. 2, 042107 (2012).

[34] D. W. Inglis, M. Lord, and R. E. Nordon, Scaling deterministic lateral displacement arrays for high throughput and dilution-free enrichment of leukocytes, J. Micromech. Microeng. 21, 054024 (2011).
[35] C. I. Civin, T. Ward, A. M. Skelley, K. Gandhi, Z. P. Lee, C. R. Dosier, J. L. D’Silva, Y. Chen, M. Kim, J. Moynihan, X. Chen, L. Aurich, S. Gulnik, G. C. Brittain, D. J. Recktenwald, R. H. Austin, and J. C. Sturm, Automated leukocyte processing by microfluidic deterministic lateral displacement, Cytom. Part A 89A, 1073 (2016).

[36] Z. Liu, F. Huang, J. Du, W. Shu, H. Feng, X. Xu, and Y. Chen, Rapid isolation of cancer cells using microfluidic deterministic lateral displacement structure, Biomicrofluidics 7, 011801 (2013).

[37] N. M. Karabacak et al., Microfluidic, marker-free isolation of circulating tumor cells from blood samples, Nat. Protoc. 9, 694 (2014).

[38] H. Okano, T. Konishi, T. Suzuki, T. Suzuki, S. Ariyasu, S. Aoki, R. Abe, and M. Hayase, Enrichment of circulating tumor cells in tumor-bearing mouse blood by a deterministic lateral displacement microfluidic device, Biomed. Microdevices 17, 59 (2015).

[39] F. Fachin et al., Monolithic chip for high-throughput blood cell depletion to sort rare circulating tumor cells, Sci. Rep. 7, 10936 (2017).

[40] R. Vernekar and T. Krüger, Breakdown of deterministic lateral displacement efficiency for non-dilute suspensions: A numerical study, Med. Eng. Phys. 37, 845 (2015).

[41] J. A. Davis, Ph.D. dissertation, Princeton University, USA, 2008.

[42] See Supplemental Material at http://link.aps.org/supplemen tal/10.1103/PhysRevApplied.12.014051 for the description of experimental and simulation methods and five supporting movies.

[43] Y. Xia, J. J. McClelland, R. Gupta, D. Qin, X.-M. Zhao, L. L. Sohn, R. J. Celotta, and G. M. Whitesides, Replica molding using polymeric materials: A practical step toward nanomanufacturing, Adv. Mater. 9, 147 (1997).

[44] P. J. Hoogerbrugge and J. M. V. A. Koelman, Simulating microscopic hydrodynamic phenomena with dissipative particle dynamics, Europhys. Lett. 19, 155 (1992).

[45] P. Espanol and P. Warren, Statistical mechanics of dissipative particle dynamics, Europhys. Lett. 30, 191 (1995).

[46] D. A. Fedosov, H. Noguchi, and G. Gompper, Multiscale modeling of blood flow: From single cells to blood rheology, Biomech. Model. Mechanobiol. 13, 239 (2014).

[47] D. Katanov, G. Gompper, and D. A. Fedosov, Microvascular blood flow resistance: Role of red blood cell migration and dispersion, Microvasc. Res. 99, 57 (2015).

[48] A. Kumar and M. D. Graham, Mechanism of Margination in Confined Flows of Blood and Other Multicomponent Suspensions, Phys. Rev. Lett. 109, 108102 (2012).

[49] K. Müller, D. A. Fedosov, and G. Gompper, Margination of micro- and nano-particles in blood flow and its effect on drug delivery, Sci. Rep. 4, 4871 (2014).

[50] E. W. Merrill, E. R. Gilliland, T. S. Lee, and E. W. Salzman, Blood rheology: Effect of fibrinogen deduced by addition, Circ. Res. 18, 437 (1966).

[51] S. Chien, S. Usami, R. J. Dellenback, and M. I. Gregersen, Shear-dependent interaction of plasma proteins with erythrocytes in blood rheology, Am. J. Physiol. 219, 143 (1970).

[52] Jülich Supercomputing Centre, JURECA: Modular supercomputer at Jülich Supercomputing Centre, J. Large-Scale Res. Facil. 4, A132 (2018). 